PSICOLOGÍA

IBEROAMERICANA
Psicología Iberoamericana ISSN: 1405-0943

revista.psicologia@ibero.mx

Universidad Iberoamericana, Ciudad de México México

\title{
Una discusión crítica del trabajo y del trabajador contemporáneo
}

Hernández Gómez, Adriana Irene

Una discusión crítica del trabajo y del trabajador contemporáneo

Psicología Iberoamericana, vol. 28, núm. 1, 2020

Universidad Iberoamericana, Ciudad de México, México

Disponible en: http://www.redalyc.org/articulo. $0 a ? i d=133963198006$ 
Artículos

\title{
Una discusión crítica del trabajo y del trabajador contemporáneo
}

\author{
A critical discussion of work and the contemporary worker \\ Adriana Irene Hernández Gómez \\ adriana.hernandez@iztacala.unam.mx \\ Universidad Nacional Autónoma de México, México
(iD) http://orcid.org/0000-0001-5174-8113 \\ Universidad Nacional Autónoma de México, México
(D) http://orcid.org/0000-0001-5174-8113
}

Psicología Iberoamericana, vol. 28, núm. 1,2020

Universidad Iberoamericana, Ciudad de México, México

Recepción: 04 Diciembre 2019 Aprobación: 19 Marzo 2020

Redalyc: http://www.redalyc.org/ articulo.oa?id=133963198006

Financiamiento

Fuente: CONACYT

No de contrato: 609148

Beneficiario: Adriana Irene Hernández Gómez

Resumen: El trabajo se ha modificado en las últimas décadas; los importantes avances científicos y tecnológicos han marcado nuevas maneras de vivir, de crear lazos y de trabajar. Estos cambios estructurales han provocado que el trabajo cada día sea más flexible, más inestable, más cambiante y más precario. Los trabajadores, a todos niveles, se enfrentan a esta situación, con una serie de consecuencias a nivel psíquico y social. El objetivo de este trabajo es presentar, por un lado, el panorama del trabajo contemporáneo y por otro lado hacer hincapié en la importancia de sostener una mirada crítica respecto de lo que acontece en el mundo de los trabajadores hoy en día. La psicología deberá detenerse sobre el análisis de las formas de organización del trabajo y sobre dichas transformaciones, de manera que la investigación e intervención en este campo se haga de manera mucho más crítica.

Palabras clave: trabajo, subjetividad, tercera revolución industrial, formas de organización del trabajo, precariedad laboral.

Abstract: Work has changed in recent decades. Important scientific and technological advances have marked new ways of living, bonding and working. These structural changes have made work more flexible, more unstable, more changing and precarious every day. Workers, at all levels, face these forms of work, with a number of consequences at the psychic and social level. The objective of this work is to present, on the one hand, the landscape of contemporary work and on the other hand to emphasize the importance of holding a critical view of what is going on in the world of work and with workers today. Psychology should dwell on the analysis of how work is formed and transforms, so that research and interventions in this field is done much more critically. Keywords: work, subjectivity, third industrial revolution, forms of work organization, precarious work.

\section{Introducción}

La concepción que se tiene de trabajo varía con la época de la que se esté hablando y, por supuesto, con el modelo económico del momento sobrevienen movimientos importantes. A lo largo de este escrito, se muestra un breve panorama histórico de la manera en que ha ido transformándose el trabajo hasta nuestros días, para posteriormente mostrar las formas de organización del trabajo contemporáneo y sus efectos psíquicos y sociales sobre los trabajadores. Discutiremos, por último, la importancia de conocer dichas transformaciones y sus efectos, de modo que la intervención psicológica en este campo se haga de manera crítica y acorde al contexto contemporáneo. 
Sabemos, por ejemplo, que en las sociedades primitivas se carecía de un lenguaje preciso para hablar de trabajo, lo cual implica que fueron sociedades estructuradas bajo linajes consanguíneos y comunitarios (Malinowski, 1986). Lo mismo sucede durante la Antigüedad, en la que el trabajo (aún sin palabra designada para él) tuvo una significación desagradable, como una labor despreciable y degradante a la que tenían que enfrentarse los esclavos. Se pensaba poco digno de personas libres desarrollar las capacidades para la obtención de ganancias, ya que la cuestión de la libertad rebasaba por mucho, la satisfacción de necesidades básicas.

Del siglo XII al siglo XVI, por ejemplo, la palabra trabajo estuvo ligada con la idea de tormento y sufrimiento, para después decantar en la noción de esfuerzo, fatiga y sacrificio, pero esta concepción fue mudando poco a poco a partir de la Edad Media, y hacia el siglo xviii a la noción de resultado útil (Plut, 2015). Weber (2007) ya explicaba que: "la empresa capitalista y el empresario capitalista son producto de los tiempos más remotos y siempre se han hallado universalmente extendidos" (p. 15). De ahí, emerge poco a poco la configuración occidental de la organización capitalista del trabajo.

Ahora bien, la entrada al siglo XIX implicará que, para poder vivir, deberán desarrollarse actividades socialmente valiosas y dignas de un pago. Además, el trabajo será considerado un valor moral en sí mismo, y no hacerlo merecerá el más severo juicio social. El descanso sólo será posible para recuperar la fuerza y la energía indispensables para realizar el trabajo; mayor tiempo de descanso se considerará holgazanería. Por supuesto, esto enmarcado en la época industrial, con la aparición de cada vez mayor número de fábricas y filas de obreros dentro de ellas. Para Bauman (2011), de este nuevo momento se esperaba "que erradicara la pobreza y garantizara la paz social. En la práctica, sirvió para entrenar y disciplinar a la gente, inculcándole la obediencia necesaria para que el nuevo régimen fabril funcionara correctamente." (p. 12).

Por otro lado, Sennett (2010) afirma que el trabajo artesanal es un bien de alto capital social, cuya transmisión de conocimientos y habilidades pasa por el cuerpo, y en el que la satisfacción del trabajo bien realizado se da por sí misma. Esto implica que el artesano le otorga sentido a su trabajo en comparación con el obrero, al que hubo que aleccionar para que continuara trabajando rutinaria y mecánicamente bajo el ideal de progreso y crecimiento continuo que no planteaba la época preindustrial. Y sin embargo, Sennett plantea también que en todo trabajo hay posibilidad de volverse artesano, ya que para él en la producción también pueden estar integrados el pensar y el sentir: el trabajo desde el cuerpo.

Ahora bien, el siglo XX se vio fuertemente marcado por la noción hegeliana del trabajo como el centro de la vida social. La producción, el hacer y las relaciones en torno al trabajo van a configurar el mundo social. Por supuesto, el fordismo será el sistema que establecerá la existencia del homo faber como centro, estableciéndose con ello una ética precisa. A su vez, las subjetividades de los trabajadores se fueron configurando en 
este sentido, construyéndose así, un sistema de sentido y significaciones a partir del trabajo, lo cual implica que el trabajo tiene efectos sobre la subjetividad. Entendemos por subjetividad el complejo entramado psíquico entre lo individual y lo colectivo.

Es así que, a partir de la década de los cincuenta del siglo pasado, comienza a gestarse una nueva configuración social, económica y política pero también subjetiva. Los grandes avances científicos y tecnológicos generan nuevas formas de trabajo, enmarcadas por la cada vez menor fuerza de la mano de obra, en pro de una enorme y acelerada producción. A esto se le conoce como la Tercera Revolución Industrial. Esta otra revolución dará paso, a su vez, a una era sin igual, la del conocimiento (Roel, 1998). En estas páginas detallaremos estos últimos cambios, en torno al trabajo y sus impactos sobre la subjetividad de los trabajadores. Consideramos a todo este conglomerado de situaciones, como ejes vitales para el estudio y comprensión del trabajador contemporáneo, desde cualquier disciplina.

\section{Primer eje: la productividad como centro y fin del trabajo}

Los cambios que hemos detallado han implicado la desaparición del estado de bienestar y con ello, importantes cambios dentro de las instituciones. Evidentemente, esto ha impactado sobre las formas en que trabajan las empresas, las cuales "han, no sólo flexibilizado sus procesos, sino multiplicado los productos[...] provocando con esto, una expansión y un crecimiento acelerado, pero también la caída de las certezas en cuanto al empleo". (Hernández, 2017, p. 14). Estos cambios han generado una cultura laboral caracterizada por la inestabilidad, la movilidad constante y, con ello, la necesidad de generar de manera continua formas de adaptación. La falta de certezas deja a los individuos en una desolación característica de nuestros tiempos.

Ahora bien, son los grandes avances tecnológicos y científicos, los que han empezado a movilizar las maneras de habitar el mundo, las formas del lazo social y, por supuesto, las formas de organización del trabajo. Hay quienes atribuyen a estos cambios un toque positivo en la vida productiva y hay quienes, con una mirada mucho más crítica, se cuestionan sobre el real beneficio de estos avances. Fernández (2006) pone en cuestionamiento esto y afirma, con un toque de ironía, que la tercera revolución industrial supone una nueva etapa en donde la productividad es el centro y eje principal. La productividad, entonces incluso a costa del trabajador.

Los cambios derivados de la tercera revolución industrial impactan la economía, la política y los intercambios globales, pero tienen además fuertes implicaciones en la vida social e individual. Estas transformaciones permean las formas de organización del trabajo y, con ello, la manera en que las personas habitan los espacios laborales, pero también sus vidas personales.

Así entramos a una nueva forma de pensar, ser, hacer y estar en el trabajo: el inicio de la etapa del toyotismo y de la del post-fordismo. 
El toyotismo es una etapa marcada por la noción de calidad, rapidez y resultado satisfactorio, que brinda grandes rendimientos para las empresas en un menor tiempo. Esto se logra sólo bajo la lógica de trabajo por equipos, mismo que desdibuja las marcadas jerarquías de las etapas anteriores, pero que compromete de manera importante al trabajador con el resultado de su propia producción (Martínez, 2010). Álvarez (2012) precisa que la efectividad de este sistema se centra en el empleo de diversos dispositivos de control para asegurar la medición de la productividad y del uso del cuerpo y del tiempo en el trabajo, pero también a través del uso de mecanismos de involucramiento (en ocasiones hasta de adoctrinamiento) del trabajador en la lógica de la producción, diríamos que se trata del perfecto sistema de construcción de sujetos productivos.

Más adelante, el post-fordismo se caracterizará por una, cada vez mayor, flexibilización del trabajo, una noción de trabajos momentáneos, de libertad y movimiento continuos. Los trabajadores han configurado una posición subjetiva de movilidad continua que les obliga a transformarse ellos mismos en una especie de producto a vender permanentemente. Son trabajadores deconectados de su labor, o bien, identificados con su labor sólo de forma momentánea. "En síntesis, una modificación del modo y la forma de la propia vida de los sujetos" (Martínez, 2010, p. 23). Desde aquí, la propuesta de Sennett (2010), para hacer del trabajo algo artesanal, resulta imposible. Hay una desconexión con el trabajo mismo, se ha dejado su valor únicamente en lo económico a través de la noción de empleo, el trabajo ya no pasa por el cuerpo.

Desde aquí es que las actuales formas de organización del trabajo, implementen diversos dispositivos de control y gestión para hacer al trabajador más productivo a cualquier costo. El modelo de dominación, entonces tuvo que ajustarse a estas nuevos requerimientos, centrándose principalmente en tres medios: la evaluación individualizada, la flexibilización y la estandarización de las tareas (Dejours, 1990, 2009, 2010, 2013, 2015, 2016; Dejours \& Bouniol, 2012). Sin embargo, estas mediciones de la productividad poco pueden decir del trabajo de las personas, la medición de la productividad deja de lado la verdadera labor, para sólo observar el producto final (Dejours, 2013), extirpando con ello, la posibilidad de apropiación e incorporación de la actividad por parte del trabajador.

\section{Segundo eje: tecnologías y trabajo}

El perfeccionamiento continuo y el uso masivo de las tecnologías de la información y la comunicación (TIC) ha generado efectos en todos sentidos (incluida la subjetividad), pero sobre todo en el hecho de que la economía se centre ahora en la información y el conocimiento. En este segundo eje, nos detendremos específicamente sobre cómo el uso de las TIC en las organizaciones transforma al trabajo, haciéndolo cada vez más flexible y cambiante.

Esta flexibilización puede representar algunas ventajas, pero también la división entre dos tipos de trabajadores: aquellos que se adaptan a 
estos cambios y se vuelven trabajadores del conocimiento, y aquellos con pocas posibilidades de adaptarse a este mercado laboral cada vez más automatizado, y por lo tanto con un futuro incierto y precario (Hormigos, 2002).

A partir de los cambios anteriormente plasmados, emergieron nuevas formas de vida, centradas en la información y en específico el conocimiento puesto al centro de las economías globales. Este hecho estructural ha generado mayor competencia por la transferencia de datos e información, y por supuesto, serán los trabajadores y su conocimiento en red lo que pondrá en marcha a esta nueva economía. Es así que surge un nuevo trabajador, el del conocimiento, también llamado por autores como Berardi (2012) como el trabajador cognitivo, emergiendo con ello el cognitariado.

De esta manera, las formas de gestionar el conocimiento por parte de las empresas, serán su principal objetivo. De aquí emerge la denominación de "recurso" o "capital" humano, y por supuesto, las nuevas formas de trabajo. Por ejemplo el teletrabajo, que por medio del uso de las TIC permitirá que el trabajador produzca donde quiera que se encuentre. Así, los discursos actuales sobre el trabajo, se basan en la noción de flexibilidad en los horarios y espacios de trabajo, ya que se vuelve cada vez menos importante la presencia física de los trabajadores. Estas nuevas formas de trabajo tienen ventajas, pero también importantes desventajas para el trabajador. Aun cuando se reducen tiempos de traslado y se incrementan las posibilidades para los trabajadores de vivir en espacios no urbanos, también se presenta una especie de invasión a la vida personal de los trabajadores, y con ello un borramiento de la línea divisoria entre tiempo de trabajo y tiempo de ocio.

Para finalizar con este eje, el uso de las TIC dentro de las organizaciones ha generado nuevos trabajadores, con todas las implicaciones sociales y subjetivas que se detallan más adelante. Se trata de un trabajador que lucha por permanecer en un espacio laboral, obedeciendo a las demandas y sometido a las formas de evaluación de la producción, un trabajador que no es consciente de la explotación en la que vive, porque cada vez más, es él mismo quien llevará el ritmo de trabajo.

Esta es la mirada que sostienen las organizaciones en sus procesos de reclutamiento, capacitación, comunicación, despidos y por supuesto, mediciones de la productividad. En pocas palabras, el trabajador perfecto hoy en día, bajo los efectos de la tercera revolución industrial y la sociedad del conocimiento, es un trabajador capaz de adaptarse a diferentes funciones, espacios y formas de trabajo. Un trabajador flexible y tal vez, en palabras de Han (2012) que se explote a sí mismo, que ya no perciba su condición de explotado, puesto que la postura consumista que describe Bauman, permite observar el paso de las formas anteriores de la explotación a una de autoexplotación y alienación de la subjetividad. 


\section{Tercer eje: efectos psíquicos y sociales del trabajo contemporáneo}

Sennett (2006) ha detallado cómo los cambios en las formas de organización del trabajo han dejado a los sujetos con una sensación de estar a la deriva, teniendo que hacer ajustes constantes para no perecer en este mundo laboral cambiante y vertiginoso. Siguiendo sus planteamientos, Bauman (2011) insiste en que la opción que ha quedado para los trabajadores, frente a esta movilidad constante, es tomar una posición de consumidor frente al trabajo, como una forma de sobrevivencia frente a esta modernidad líquida, esta continua desterritorialización, planteará Berardi (2015). Hernández (2012, 2017) ha insistido en que los trabajadores en la actualidad se encuentran luchando entre dos puntos contradictorios: la demanda de progreso y desarrollo (ligado a la pertenencia a un espacio laboral) y, por otro lado, a la demanda de movilidad constante y flexibilidad, que establece una negativa a "echar raíces" en un espacio laboral. Estas contradicciones, sumadas a las formas actuales de gestión y control laboral, han precarizado el trabajo y han hecho su vivencia cada vez más compleja.

A su vez, Rolo (2015) afirma que estos cambios implican un debilitamiento de los derechos sociales, con sus obvios efectos deletéreos sobre la salud. Los trabajadores parecen estar a merced del mercado, de sus cambios y sus demandas. La investigación de Rolo muestra cómo el trabajador se ve forzado muchas veces a emplear la mentira como medio para cumplir con lo que se le demanda; dicha posición lo deja en un sufrimiento ético, o bien en una postura indiferente frente al otro. Las formas de organización del trabajo, cada vez más, crean sistemas de sumisión y de banalización de la injusticia (Dejours, 2009), lo cual implica, a su vez, la ruptura del lazo social, de la posibilidad de hacer comunidad y, en ese sentido el imperio del individualismo que deja a los trabajadores sobreviviendo en solitario.

De esta forma, será la flexibilización del trabajo derivada de los procesos de globalización, enmarcados por la denominada tercera revolución industrial, la que permite observar las nuevas formas de trabajo y los nuevos tipos de contratación (temporales, por honorarios, por empresas externas, sin prestaciones, sin horarios establecidos, etcétera) usados como estrategias empresariales precisas para obtener mayores rendimientos. Esta continua flexibilización lleva a la aparición del autoempleo y el emprendedurismo como alternativas posibles para los trabajadores hoy en día frente a la precarización del trabajo. Es el mayor número de empleos actualmente en el sector servicios, el que permite poner en la mira a la producción inmaterial, pero también el trabajo cognitivo.

El trabajador cognitivo, el que no puede saber de su trabajo, más que a través de las evaluaciones individualizadas del desempeño y la producción, pareciera estar psíquica y corporalmente desconectado de su trabajo. Berardi (2015) ha mostrado los efectos nocivos de esta desconexión del trabajador con su tarea, pero ha sido especialmente la psicodinámica del trabajo la que ha puesto énfasis en los efectos que 
tienen sobre el psiquismo estas transformaciones (Dejours, 2009, 2010, 2013, 2015, 2016; Dejours \& Bouniol, 2012; Duarte, 2017; Rolo, 2015). Estos efectos sobrepasan los conocidos estrés y síndrome de burnout tan abordados por la psicología organizacional. Dejours y Gernet (2014) han detallado ampliamente cómo se presentan los casos de depresión, psicosis, patologías postraumáticas, los efectos de la violencia en el trabajo, del acoso sexual y moral, las patologías de la sobrecarga, las adicciones, los intentos de suicidio y suicidios en el trabajo. Estos autores han concluido que no son situaciones que deban pensarse desde lo individual, sino específicamente en consideración de lo colectivo y lo organizacional. Esto quiere decir que son las formas de organización del trabajo las que llevan a los trabajadores a este tipo de manifestaciones psicopatológicas.

Adicional a esto, un efecto de estos profundos cambios, es la incorporación de un mayor número de mujeres al mercado laboral, lo cual supone muchas ventajas para el mercado y para las mujeres mismas, pero también implica la necesaria adecuación de las condiciones de trabajo para ellas. El trabajo para las mujeres se duplica y triplica; a su trabajo no remunerado de cuidados y crianza, se le añaden extensas jornadas de trabajo, al cual no pueden negarse, ya que quedarían fuera de esta injusta contienda. Esto último requiere, por supuesto, un extenso análisis por sí mismo, pero al sólo enunciarlo, se hace evidente la aparición o acentuación de problemáticas emocionales, físicas, familiares y sociales, que derivan de esta sobrecarga, la cual se acentúa con la falta de reconocimiento de la labor efectuada, sobre todo en los hogares y con la carencia de estrategias precisas para la incorporación de mujeres madres de familia al mercado laboral, así como de la equidad respecto de las condiciones de trabajo para ellas.

Para cerrar este eje, es importante mencionar que los trabajadores no son pasivos frente a estos cambios, sino que toman postura frente a ellos. Como hemos mencionado, estas posturas varían del miedo y el aburrimiento, la sumisión y la obediencia, atravesando por las más diversas formas defensivas hasta las acciones concretas de resistencia (Dejours, 2013; Duarte, 2017), sin olvidar algunas posiciones que toman ventaja de estas transformaciones.

\section{Conclusiones}

Es imposible pensar el trabajo sin hacer un minucioso análisis de las condiciones sociales, económicas y políticas que se gestan en cada momento de la historia. Y como revisamos a lo largo de este escrito, todas las transformaciones han implicado nuevas formas en la división técnica del trabajo, en la prescripción de tareas y sobre todo en las formas de gestión y de gobierno (management) al interior de los lugares de trabajo (Dejours, 1990, 2009, 2010, 2013, 2015, 2016; Dejours \& Bouniol, 2012). Por supuesto, los trabajadores, en todos los sectores y a todos los niveles, se ven impactos por estas modificaciones.

Así, el trabajo se ha vuelto cada vez más flexible e inestable, lo cual implica que las certezas del trabajo para toda la vida no son ya viables, 
como lo fue para otras generaciones. De esta manera, surgen nuevas formas de contratación, además de la posibilidad del teletrabajo y con ello la disminución de costos para las empresas (ahora a cargo del empleado). Todos los procesos de evaluación de la productividad del trabajador están establecidos por medidas controladas por tecnologías diversas; la precarización del trabajo se hace más evidente, en términos de sueldos, horas de trabajo, y, por supuesto, salud física y emocional. Con las transformaciones que trajo la expansión y uso masivo de las TIC en las organizaciones laborales, todos sus procesos se centran sobre el manejo, almacenamiento y control de la información por medio de dichas tecnologías, por lo que el conocimiento será el eje central de las economías globales y desde aquí se deciden todos los procesos. Los llamados recursos humanos, el capital humano se buscará siguiendo una línea clara: trabajadores con conocimiento preciso, trabajadores adaptables, flexibles y dispuestos a no pensar en el futuro a largo plazo.

Finalmente, ¿cuál es la posición de la psicología frente al estudio del trabajo? La psicología organizacional poco se ha ocupado de las circunstancias nocivas que viven los trabajadores hoy en día y se ha centrado en buscar que estos se adapten de mejor forma a los cambios descritos anteriormente. Esto contribuye a la formación de un malestar psíquico y social cada día más evidente. Es vital que la psicología repare en que las actuales formas de organización del trabajo construyen trabajadores "perfectos" para las empresas, aquellos que asumen sin más el lugar que se les otorga, y bajo las condiciones más precarias. Es necesario que la psicología, en este terreno, recupere su posición crítica y sea consciente de las transformaciones que el trabajo ha tenido a lo largo del tiempo. Investigar o intervenir en el campo del trabajo, desde la psicología, no puede hacerse sin tomar en cuenta las cuestiones estructurales que subyacen a los problemas del trabajo y los trabajadores. Esta posición crítica debe considerar al menos estos tres ejes que aquí proponemos: la productividad a toda costa, la inserción de las tecnologías al mundo del trabajo y sus efectos psíquicos y sociales.

\section{Agradecimientos}

Este trabajo forma parte de la investigación doctoral: "Malestar subjetivo y estrategias de resistencia en altos ejecutivos: Análisis de las formas contemporáneas de organización del trabajo", dirigida por el Dr. Fernando Manuel González y González y contó con el apoyo de CONACYT, No becario: 609148.

\section{Referencias}

Álvarez, D. (2012). El toyotismo como sistema de flexibilización de la fuerza de trabajo. Una mirada desde la construcción de la productividad en los trabajadores de la fábrica japonesa (1994-2005). Si Somos Americanos. Revista de Estudios Transfronterizos, 12(2), 181-201. http://dx.doi.org/1 $0.4067 /$ S0719-09482012000200008 
Bauman, Z. (2011). Trabajo, consumismo y nuevos pobres (V. de los Ángeles Boschiroli, trad.). Gedisa.

Berardi, F. (2012). El alma y el trabajo (F. Cassalter \& P. Pagliai, trad.). Elefanta Editorial.

Berardi, F. (2015). La fábrica de la infelicidad. Nuevas formas de trabajo y movimiento global (M. Aguilar Hendrikson y P. Amigot Leatxe, trads.). Traficantes de sueños.

Dejours, C. (1990). Trabajo y desgaste mental: una contribución a la psicopatologia del trabajo (P. Neffa y G. Neffa, trad.). Hvmanitas.

Dejours, C. (2009). Trabajo y sufrimiento: cuando la injusticia se hace banal (B. Díez, trad.). Modus Laborandi.

Dejours, C. (2010). Nouvelles formes de servitude et suicide. En C. Dejours (Ed.), Obsevations cliniques en psychopathologie du travail (pp. 131-162). Presses Universitaires de France.

Dejours, C. (2013). Travail vivant 1: sexualité et travail. Éditions Payot \& Rivages.

Dejours, C. (2015). Travail. Usure mentale: essai de psychopathologie du travail. Bayard.

Dejours, C. (2016). Situations du travail. Presses Universitaires de France.

Dejours, C., \& Bouniol, B. (2012). La panne: repenser le travail et changer la vie. Bayard.

Dejours, C., \& Gernet, I. (2014). Psicopatología del trabajo (G. Neffa, Trad.). Miño y Dávila.

Duarte, A. (2017). Défenses et résistences en psychodynamique du travail (Tesis doctoral). Université Paris Descartes, Francia. https://doi.org/10.13140/ RG.2.2.29280.33289

Fernández, O. (2006). ¿ ¿Tercera revolución industrial? Reflexiones desde la lógica del "empisimo convencional". Economía y Desarrollo, 140(2), 3859.

Han, B. C. (2012). La sociedad del cansancio (A. Saratxaga Arregi, Trad.). Herder.

Hernández, A. (2012). El cuerpo en la época de la producción posmaterial. Metapolitica, 19(79), 46-50.

Hernández, A. (2017). Subjetividad y trabajo. Pensar al sujeto en las formas del trabajo contemporáneo. Metapolitica, 21(97), 14-25.

Hormigos, J. (2002). Nuevas formas de trabajo en la sociedad conocimiento: el teletrabajo. Revista Castellano-Manchega En Ciencias Sociales, 1(5), $213-$ 226. http://dx.doi.org/10.20932/barataria.v0i5.268

Malinowski, B. (1986). Los argonautas del pacifico norte (A. J. Desmonts, trad.). Planeta de Agostini.

Martínez, C. (2010). Psicología social en las organizaciones: estrategias, tácticas y técnicas para el cambio organizacional. Lugar Editorial.

Plut, S. (2015). Trabajo y subjetividad. Estudios psicoanaliticas. Psicolibro Ediciones.

Roel, V. (1998). La tercera revolución industrial y la era del conocimiento. Fondo Editorial UNSMN.

Rolo, D. (2015). Mentir au travail. Presses Universitaires de France. 
Sennett, R. (2006). La corrosión del carácter. Las consecuencias personales del trabajo en el nuevo capitalismo (D. Najmías, trad.). Anagrama.

Sennett, R. (2010). El artesano (M. A. Galmarini, trad.). Anagrama.

Weber, M. (2007). La ética protestante y el espiritu del capitalismo (L. Legaz Lacambra, trad.). Colofón. 